\title{
Suspected tumor-to-meningioma metastasis: A case report
}

\author{
IURI SANTANA NEVILLE, DAVI FONTOURA SOLLA, ARTHUR MAYNART OLIVEIRA, \\ CESAR CASAROLLI, MANOEL JACOBSEN TEIXEIRA and WELLINGSON SILVA PAIVA
}

Division of Neurological Surgery, University of Sao Paulo Medical School, Sao Paulo, SP 05403010, Brazil

Received May 22, 2015; Accepted April 22, 2016

DOI: $10.3892 / 01.2017 .5655$

\begin{abstract}
Tumor-to-meningioma metastasis (TMM) is a fairly uncommon phenomenon. Only 7 cases of prostate cancer with TMM have previously been described in the literature. The present study aimed to report a case of prostate cancer TMM, and to discuss the relevant clinical and neuroimaging aspects of this condition. A 68-year-old patient presented with headaches, poor visual acuity in the left eye and ipsilateral eyelid droop 3 years after a Simpson II resection of a left sphenoid wing meningioma. Computed tomography revealed a hyperdense area suggestive of a recurrent left sphenoid wing meningioma. During microsurgical resection of tumor, the tumor presented a fibrous aspect and bled profusely. In the histological examination, a metastatic adenocarcinoma was identified inside the transitional meningioma. The immunohistochemical exam favored a prostatic primary site. The patient died two months later of septic shock from pneumonia. This is a rare metastatic presentation. The pre-operative diagnosis of TMM remains challenging in the majority of cases.
\end{abstract}

\section{Introduction}

Tumor-to-meningioma metastasis (TMM) is a fairly uncommon phenomenon, with just over 100 cases published to date since the first case reported by Fried in 1930 (1,2). Although meningiomas have been reported as the most common intracranial neoplasms to harbor metastasis, any benign or malignant tumor can be a recipient (3-6). Despite certain radiological features being suggestive of the diagnosis of TMM, such as atypical signal changes in MRI suggesting the presence of another tumor within a meningioma, they are neither sensitive nor specific, making the pre-surgical diagnosis extremely difficult $(7,8)$. The prevalence of TMM increases with age and apparently there is no gender difference. Only 7 cases of prostate cancer with TMM have previously been described in

Correspondence to: Professor Wellingson Silva Paiva, Division of Neurological Surgery, University of Sao Paulo Medical School, 255 Eneas Aguiar Street, Office 4079, Sao Paulo, SP 05403010, Brazil E-mail: wellingsonpaiva@yahoo.com.br

Key words: meningioma, adenocarcinoma, neuroimaging, pathology, prostate, cancer the literature $(6,9-13)$. The present study aimed to report a case of prostate cancer TMM, and to discuss the relevant clinical and neuroimaging aspects of this condition.

\section{Case report}

A 68-year-old male with a diagnosis of left sphenoid wing meningioma, observed on brain magnetic resonance imagin (1.5T device; Philips Medical Systems, Inc., Bothell, WA, USA), underwent a Simpson II resection meningioma at Hospital das Clínicas, University of Sao Paulo (Sao Paulo, Brazil) (Fig. 1). The patient experienced a good post-operative clinical course and there were no signs of tumor recurrence on imaging. However, 3 years after the resection, the patient was admitted to the Emergency Department of the same hospital complaining of headaches, poor visual acuity in the left eye and ipsilateral eyelid droop. These symptoms were present for 1 month. A neurological exam at admission revealed normal levels of consciousness and cognition, with no sensory or motor deficits. Upon cranial nerve evaluation, there was impairment of the visual acuity on the left side, associated with ipsilateral oculomotor nerve palsy. The visual fields were preserved and no other cranial nerve was altered.

Head computed tomography (General Electric, Milwaukee, WI, USA) revealed a hyperdense area suggestive of a recurrent left sphenoid wing meningioma (Fig. 2). Gross total resection was performed through the previous craniectomy access. A large gliotic area with a cyst and enlarged subarachnoid space facilitated access to the lesion. The tumor presented a fibrous aspect and bled profusely. The tumor adhered to the middle fossa and left sphenoid wing, and was resected with no complications. The intraoperative tumor aspect was suggestive of a meningioma: A well-circumscribed yellowish extra-axial lesion attached to dura-matter. Post-operative axial head computed tomography (CT) scan was performed and showed complete tumor removal (Fig. 3). Due to septic shock from pneumonia on postoperative course and the patient succumbing to the disease two months after surgery, systemic cancer therapy was not performed.

In the histological examination, the tumor was identified as a metastatic adenocarcinoma inside a transitional meningioma (Fig. 4). The surgical specimen consisted of brownish tumor fragments totaling $4.2 \times 3.8 \times 0.8 \mathrm{~cm}$ with fibroelastic consistency. All the fragments underwent fixation with formalin and histological examination. The immunohistochemical examination favored a prostatic primary site, with the following results: Negative reactivity for caudal-type homeobox-2, 


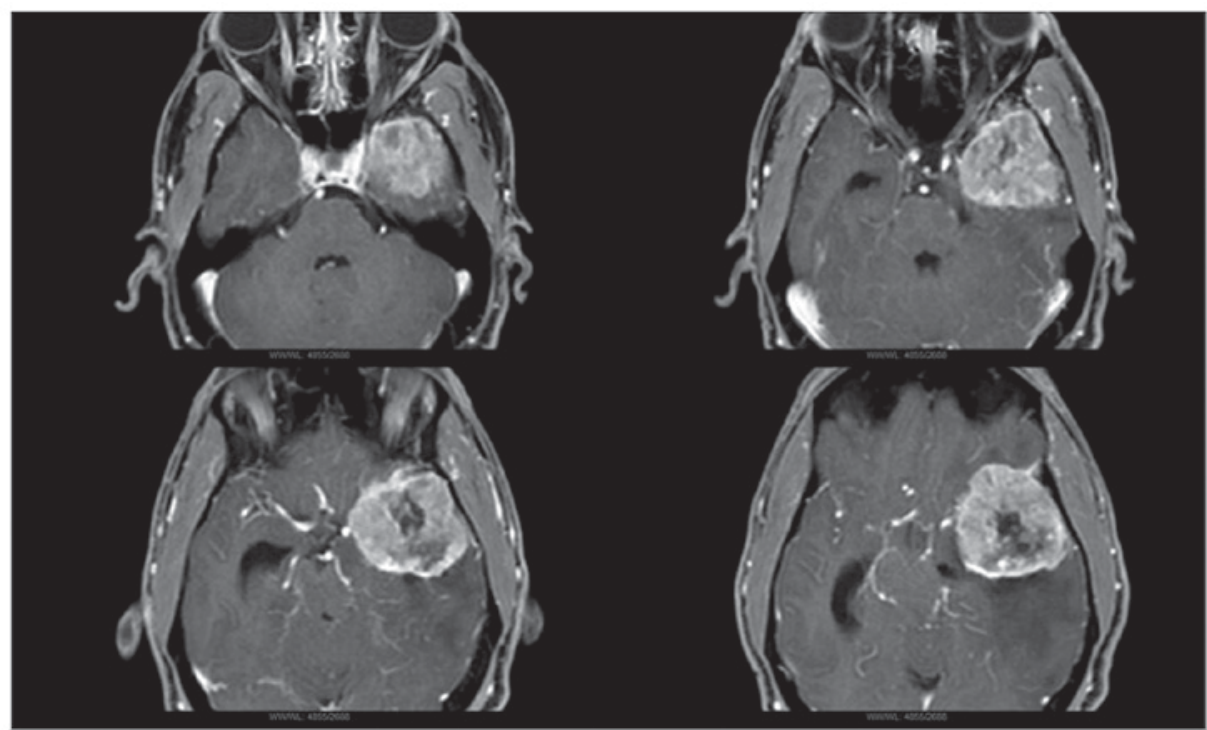

Figure 1. Initial presentation (2008). Brain T1-weighted post-contrast axial magnetic resonance imaging showing an enhancing dural-based mass occupying the left anterior inferior temporal fossa surrounded by moderate vasogenic edema and a mass effect. The mass exhibited an intimate association with the underlying left sphenoid wing.

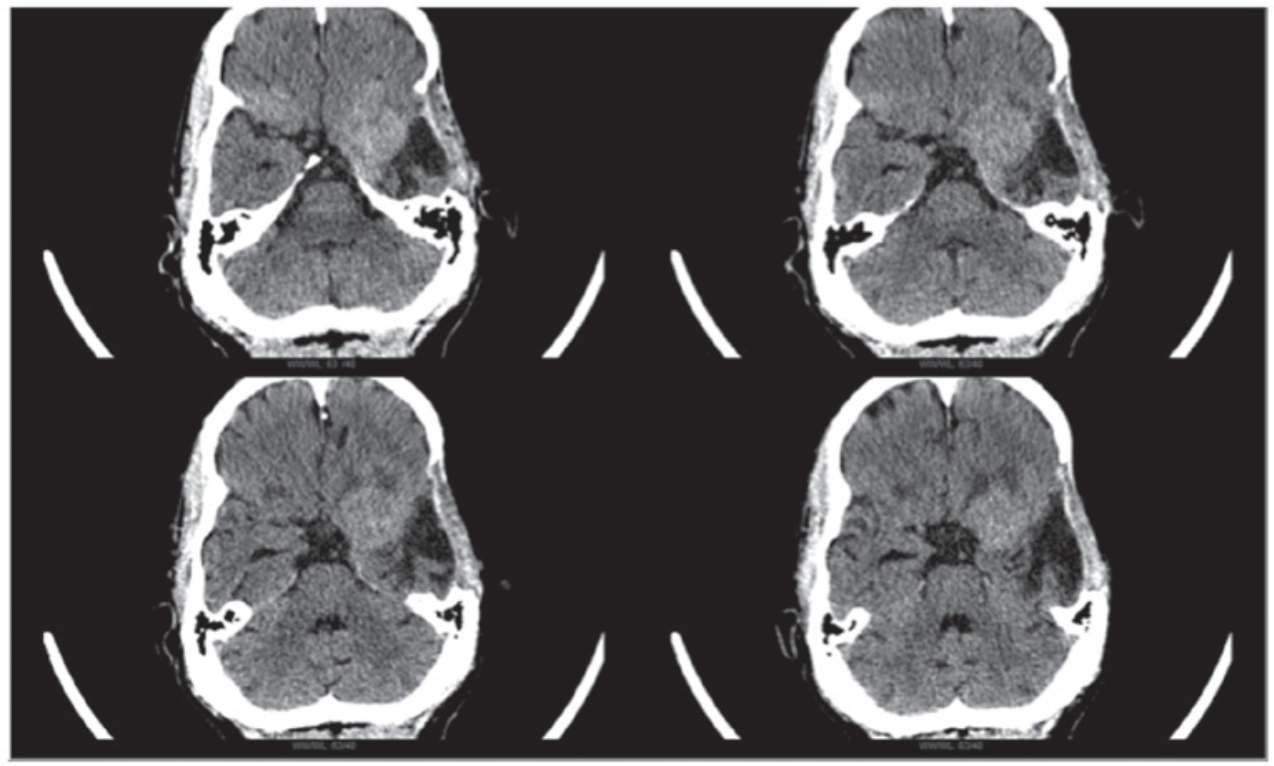

Figure 2. Computed axial tomography on readmission in 2011. A hyperdense area encompassed by mild cerebral edema was suggestive of recurrent left sphenoid wing meningioma. There was no relevant mass effect and a large gliotic area with an enlarged liquor space compatible with the prior procedure was also observed.

cytokeratin 20 , cytokeratin 7 , thyroid transcription factor-1 and vimentin, and positivity for epithelial membrane antigen, prostatic acid phosphatase and prostate-specific antigen (PSA). Subsequent investigation revealed a PSA level of $1,295 \mathrm{ng} / \mathrm{ml}$ (reference value, $<4.0 \mathrm{ng} / \mathrm{ml}$ ) and signs of advanced metastatic prostate disease on cancer staging.

\section{Discussion}

Prostate cancer TMM is a rare condition. To the best of our knowledge, the present case is only the eighth case reported in the literature. Table I summarizes all the cases to date. The most commonly encountered malignant donor tumor is

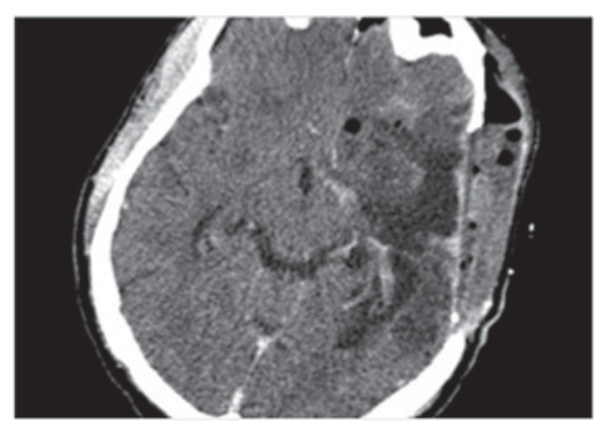

Figure 3. Post-operative axial computed tomography showing the final aspect after the new resection. Beyond the gliosis, certain heterogeneous hypodense areas and residual pneumocephalus were apparent. 


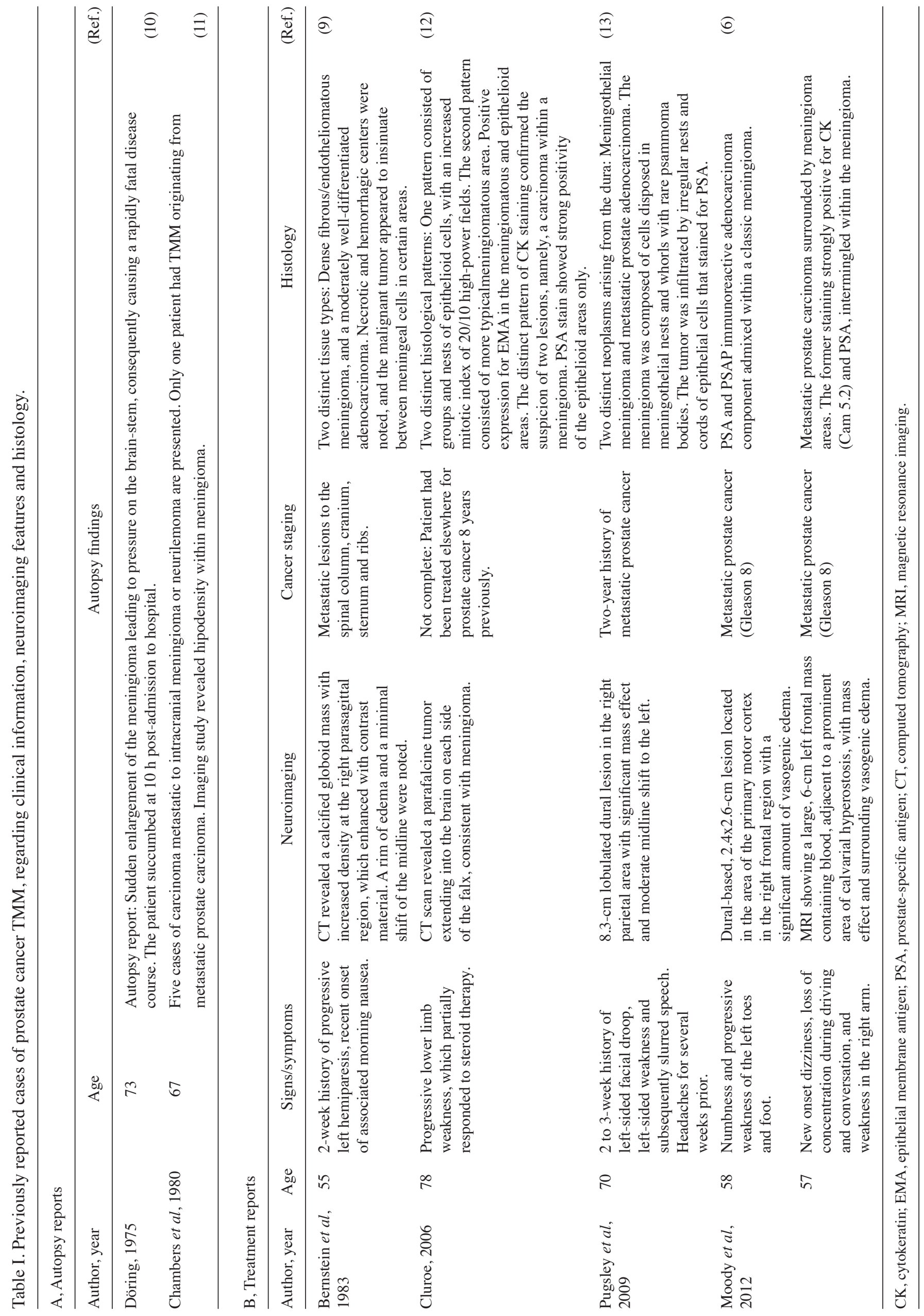


Table II. Epidemiological and pathophysiological mechanisms suggested for the predilection of meningioma to harbor metastasis.

Proposed mechanism

(Ref.)

High incidence of meningioma among intracranial neoplasms (15)

Increase in incidence with age, as occurs with metastasis

Benign and slow growth rate

Hypervascularity

High collagen content

High lipid content

High expression of cell adhesion molecules, such as E-cadherin(15)

Overexpression of oncogenes (e.g., c-Myc)

Hormonal factors (estrogen and progesteron receptors)

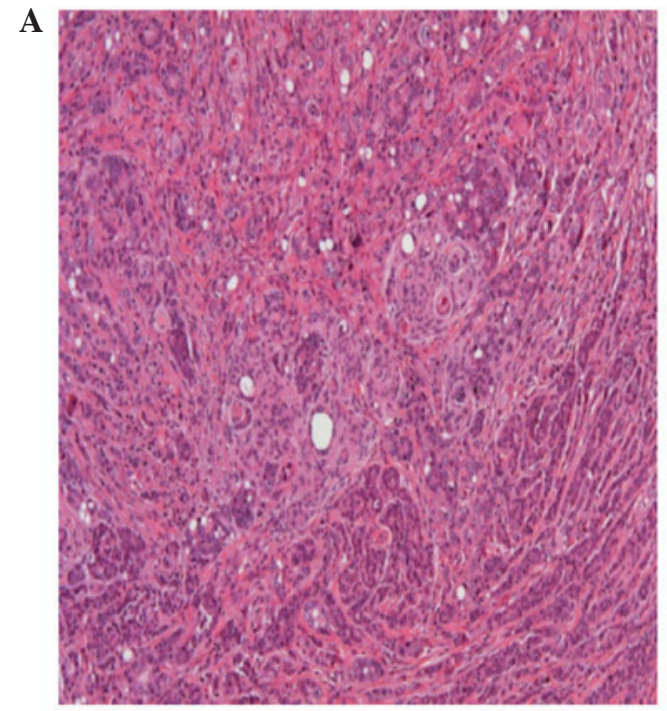

B

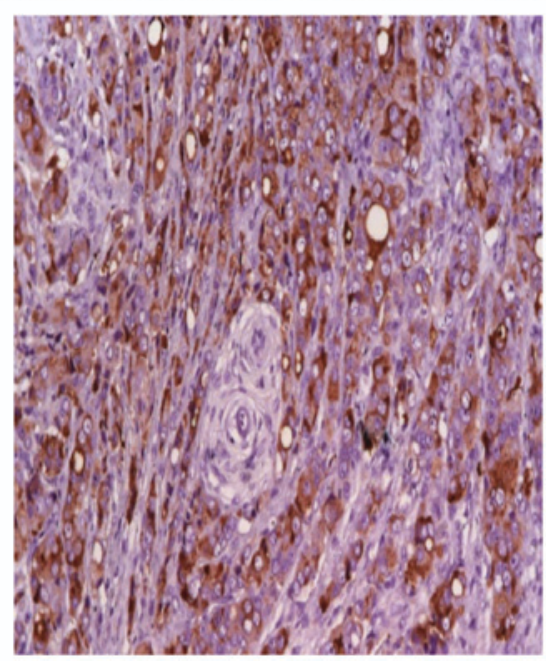

Figure 4. (A) Histological analysis. In metastasis of prostate adenocarcinoma into transitional meningioma. Malignant neoplasm composed of basophilic glandular structures (lower region) infiltrating meningioma with characteristic swirls (center) (hematoxylin and eosin staining; magnification, x100). (B) Immunohistochemistry for prostate-specific acid phosphatase. Positivity in adenocarcinoma around negative cells nest, typical of meningioma (magnification, $\mathrm{x} 200$ )

lung adenocarcinoma in males and breast adenocarcinoma in females, the latter mostly arising in women in their fifth and sixth decades of life $(7,8)$. In a recent review of the literature (2), 114 cases of TMM were reported, with 54 breast cancer cases and 23 lung cancer cases, representing 47.4 and $20.2 \%$ of the total, respectively.

Although cerebral metastases are by far the most common intracranial tumors, the occurrence of TMM is still considered a rare condition (2). According to a study by Barz, among 8,371 autopsies, 4 cases $(0.05 \%)$ of a carcinoma metastasizing into a meningioma were found (14). Notably, the yielding of less common primary sites of the genitourinary system to such metastasis has been reported, including in the kidneys and prostate (2).

Epidemiological and pathophysiological mechanisms have been proposed to explain the meningioma's higher propensity to harbor extracranial metastatic lesions. Table II outlines the various reasons proposed. Apparently, a combination of epidemiological aspects and the biology of meningiomas explain, at least in part, this association. Epidemiological studies have shown that metastatic cancer and meningioma occur simultaneously with high frequencies during aging (15). The benign and slow growth rate associated with high collagen and lipid content, characteristic elements of meningiomas, render a favorable, non-competitive environment for metastasis to develop (16). Another important point concerns the hypervascularity of meningiomas, which may enhance the chances of the meningioma to receive hematogenous metastasis $(4,17)$. In cases of breast cancer TMM, two relevant factors have been postulated to play a role in metastatic seeding: i) High expression of cell adhesion molecules, such as E-cadherin $(18,19)$, and ii) hormonal factors, particularly estrogen and progesterone receptor expression (20,21). Lastly, overexpression of oncogenes in meningioma and metastatic carcinoma could contribute to the simultaneous occurrence $(22,23)$.

It is crucial to differentiate TMM from the occurrence of collision tumors, which are due to the growth of two isolated tumors that successively collide (24). In order to discriminate between these two conditions, in 1968, Campbell et al (25) proposed four criteria for the diagnosis of tumor-to-tumor metastasis, while in 1984, Pamphlett (26) depicted two diagnostic criteria for TMM: i) At least partial enclosure of the metastatic focus by a rim of benign histologically distinct host tumor tissue; and ii) the proven existence of metastasizing primary carcinoma compatible with the metastasis. The present case fulfills these criteria.

Even as the second most common cancer worldwide, prostate cancer rarely metastasizes to the brain, with only $0.2-0.6 \%$ of such cases $(9,27-29)$. In general, central nervous system involvement is restricted to the spine, involving the perineural and capsular lymphatic system, as well as Batson's venous plexus (29). Furthermore, certain studies have reported dural metastasis from prostate cancer simulating meningiomas (30-34). However, the present case represents a distinct entity: The metastatic prostate cancer is surrounded by the recipient, benign tumor (meningioma), meeting the aforementioned criteria (26) for 'true' TMM.

The present case has another distinctive feature in that the TMM was the first clinical manifestation of an occult primary prostate cancer. The histology report triggered a systemic investigation, leading to the diagnosis of disseminated cancer. However, the majority of the TMM cases reported to date 
Table III. Neuroimaging features frequently associated with TMM.

\begin{tabular}{ll}
\hline Diagnostic technique & \multicolumn{1}{c}{ Feature } \\
\hline CT & $\begin{array}{l}\text { Hyperdense lesion } \\
\text { Hypodense lesion (when associated with necrosis) }\end{array}$ \\
$\begin{array}{l}\text { MRI } \\
\text { Routine MRI } \\
\text { pMRI }\end{array}$ & $\begin{array}{l}\text { Atypical signal changes suggesting the presence of another tumor within a meningioma. } \\
\text { Marked increase in rCBV within a tumor. May reveal different curve characteristics suggesting } \\
\text { distinct intratumoral vascular properties. }\end{array}$ \\
MRS & $\begin{array}{l}\text { Increase in alanine/Cr ratio is highly suggestive of a meningioma. A decrease in NAA, a prominent } \\
\text { increase in Cho and lactate lipid peaks, and a high lipid/Cr ratio favor metastasis. }\end{array}$ \\
PET-CT & FDG may play a role in the pre-operative diagnosis of TMM. An increase in T:N ratio may be observed. \\
\hline CT, computed tomography; MRI, magnetic resonance imagimg; pMRI, perfusion-MRI; rCBV, regional cerebral blood volume; MRS, magnetic reso- \\
nance spectroscopy; PET-CT, positron emission tomography-CT; Cr, creatine; Cho, choline; FDG, fluorodeoxyglucose; NAA, n-acetyl aspartate; T:N \\
ratio, tumor-to-normal ratio; TMM, tumor-to-meningioma metastasis.
\end{tabular}

had a previous diagnosis of metastatic disease, rendering a pre-surgical hypothesis of TMM $(6,7,35)$. Conversely, Caroli et al (8) reported 3 cases of TMM in patients with occult cancer. Determining the occurrence of TMM in a patient without a previous history of metastatic disease remains a major challenge.

Clinical and neuroimaging features may be pivotal in the pre-operative suspicion of TMM. From the clinical point of view, three factors commonly associated with TMM were identified in the present study: i) A previously known history of metastatic disease, ii) uncontrolled systemic disease and iii) a rapid deterioration of neurological condition. However, none of these factors are specific for TMM, making a pre-operative diagnosis unlikely. Besides clinical information, neuroimaging may be extremely useful for assessing these patients. Table III highlights the most notable characteristics associated with TMM. CT findings are described as either a hyperdense or hypodense (when associated with necrosis) area, which does not provide much information. However, atypical changes of signal on routine MRI can suggest the coexistence of two different tumors $(6,8)$. The use of specialized, physiology-based neuroimaging modalities, such as perfusion MRI (pMRI), magnetic resonance spectroscopy and positron-emission tomography-CT (PET-CT), may result in a superior diagnostic yield for this condition $(7,8)$. pMRI can potentially reveal two different curve characteristics of regional cerebral blood volume, suggesting different intratumoral vascular properties (36). When differentiating malignant tumors from benign tumors, $\mathrm{N}$-acetylaspartate (NAA)/choline (Cho), NAA/Cho + creatine $(\mathrm{Cr})$, lactate/Cr and alanine/Cr ratios are useful. Increased lipid/ $\mathrm{Cr}$ and alanine/Cr ratios may enable metastasis and meningiomas to be distinguished from other tumors. The most striking characteristics of metastasis are a decrease in NAA, a prominent increase in Cho and lactate lipid peaks, and a high lipid/Cr ratio (37). Fukushima et al (38) used PET-CT with fluorodeoxyglucose and the positron-emitting radioactive isotope fluorine-18 in a patient with lung adenocarcinoma metastasizing to vestibular schwannoma that was suspected on pre-operative imaging studies. Despite this not being a meningioma case, the rationale of using a neuroimaging technique to assess the metabolic pattern of a tumor may be applied for tumor-to-tumor metastasis (TTM) cases. The tumor-to-normal ratio, one of the parameters used, was markedly high, which offered corroborating evidence of TMM.

The pre-operative high suspicion of TMM can modify the treatment of TMM when compared with routine meningioma excisions. Taking into consideration the possibility of TMM, surgeons may adopt different surgical approaches. Likewise, pathologists may look differently and systematically at the entire tumor, reducing the chance of misdiagnosis if the TMM hypothesis was not taken into account (7).

The present study is a case report of a patient with a more likely preoperative diagnosis of a benign tumor based on imaging studies that had a metastatic prostate cancer with unfavorable postoperative course. In such cases, physicians caring for patients with brain tumors should be familiar with TMMs, since the preoperative diagnosis remains challenging for the majority of cases. Clinical and neuroimaging features are crucial for this purpose, giving further consideration to this hypothesis. However, clinical and radiological findings are neither sensitive nor specific for TMM diagnosis, and being subjected to histopathological study is the only definitive diagnostic method. Finally, the effort of forming a preoperative suspicion can be rewarding, allowing the more appropriate conduct of the professionals involved with the patient care.

\section{References}

1. Fried BM: Metastatic inoculation of a meningioma by cancer cells from a bronchiogenic carcinoma. Am J Pathol 6: 47-52.1, 1930.

2. Erdogan H, Aydin MV and Tasdemiroglu E: Tumor-to-tumor metastasis of the central nervous system. Turk Neurosurg 24: 151-162, 2014.

3. Petraki C, Vaslamatzis M, Argyrakos T, Petraki K, Strataki M, Alexopoulos C and Sotsiou F: Tumor to tumor metastasis: Report of two cases and review of the literature. Int J Surg Pathol 11: $127-135,2003$.

4. Lanotte M, Benech F, Panciani PP, Cassoni P and Ducati A: Systemic cancer metastasis in a meningioma: Report of two cases and review of the literature. Clin Neurol Neurosurg 111: 87-93, 2009.

5. Schmitt HP: Metastases of malignant neoplasms to intracranial tumours: The 'tumour-in-a-tumour' phenomenon. Virchows Arch A Pathol Anat Histopathol 405: 155-160, 1984.

6. Moody P, Murtagh K, Piduru S, Brem S, Murtagh R and Rojani AM: Tumor-to-tumor metastasis: Pathology and neuroimaging considerations. Int J Clin Exp Pathol 5: 367-373, 2012. 
7. Sayegh ET, Henderson GA, Burch EA, Reis GF, Cha S, Oh T, Bloch $\mathrm{O}$ and Parsa AT: Intrameningioma metastasis of breast carcinoma. Rare Tumors 6: 5313, 2014.

8. Caroli E, Salvati M, Giangaspero F, Ferrante L and Santoro A: Intrameningioma metastasis as first clinical manifestation of occult primary breast carcinoma. Neurosurg Rev 29: 49-54, 2006.

9. Bernstein RA, Grumet KA and Wetzel N: Metastasis of prostatic carcinoma to intracranial meningioma. Case report. J Neurosurg 58: 774-777, 1983

10. Döring L: Metastasis of carcinoma of prostate to meningioma. Virchows Arch A Pathol Anat Histol 366: 87-91, 1975.

11. Chambers PW, Davis RL, Blanding JD and Buck FS: Metastases to primary intracranial meningiomas and neurilemomas. Arch Pathol Lab Med 104: 350-354, 1980.

12. Cluroe AD: Metastasis to meningioma: Clues and investigation. Pathology 38: 76-78, 2006.

13. Pugsley D, Bailly G, Gupta R, Wilke D and Wood L: A case of metastatic adenocarcinoma of the prostate arising in a meningioma. Can Urol Assoc J 3: E4-E6, 2009.

14. Barz $\mathrm{H}$ : The incidence of metastatic carcinomas in meningiomas. A report of 4 cases. Zentralbl Allg Pathol 127: 367-374, 1983 (In German)

15. Schoenberg BS, Christine BW and Whisnant JP: Nervous system neoplasms and primary malignancies of other sites. The unique association between meningiomas and breast cancer. Neurology 25: 705-712, 1975.

16. Richardson JF and Katayama I: Neoplasm to neoplasm metastasis. An acidophil adenoma harbouring metastatic carcinoma: A case report. Arch Pathol 91: 135-139, 1971

17. Best PV: Metastatic carcinoma in a meningioma: Report of a case. J Neurosurg 20, 892-894, 1963.

18. Watanabe T, Fujisawa H, Hasegawa M, Arakawa Y, Yamashita J, Ueda F and Suzuki M: Metastasis of breast cancer to intracranial meningioma: Case report. Am J Clin Oncol 25: 414-417, 2002.

19. Shimada S, Ishizawa $K$ and Hirose T: Expression of E-cadherin and catenins in meningioma: Ubiquitous expression and its irrelevance to malignancy. Pathol Int 55: 1-7, 2005.

20. Doyle S, Messiou C, Rutherford JM and Dineen RA: Cancer presenting during pregnancy: Radiological perspectives. Clin Radiol 64: 857-871, 2009.

21. Grann VR, Troxel AB, Zojwalla NJ, Jacobson JS, Hershman D and Neugut AI: Hormone receptor status and survival in a population-based cohort of patients with breast carcinoma. Cancer 103: 2241-2251, 2005.

22. Kozbor D and Croce CM: Amplification of the c-myc oncogene in one of five human breast carcinoma cell lines. Cancer Res 44 438-441, 1984.

23. Elmaci L, Ekinci G, Kurtkaya O, Sav A and Pamir MN: Tumor in tumor: Metastasis of breast carcinoma to intracranial meningioma. Tumori 87: 423-427, 2001.
24. Anlyan FH, Heinzen BR and Carras R: Metastasis of tumor to second different tumor: Collision tumors. JAMA 212: 2124, 1970.

25. Campbell LV Jr, Gilbert E, Chamberlain CR Jr and Watne AL: Metastases of cancer to cancer. Cancer 22: 635-643, 1968.

26. Pamphlett R: Carcinoma metastasis to meningioma. J Neurol Neurosurg Psychiatry 47: 561-563, 1984.

27. Lynes WL, Bostwick DG, Freiha FS and Stamey TA: Parenchymal brain metastases from adenocarcinoma of prostate. Urology 28: 280-287, 1986.

28. Tremont-Lukats IW: Brain metastasis from prostate carcinoma: The M.D. Anderson cancer center experience. Cancer 98 363-368, 2003

29. Catane R, Kaufman J, West C, Merrin C, Tsukada Y and Murphy GP: Brain metastasis from prostatic carcinoma. Cancer 38: 2583-2587, 1976.

30. Lyons MK, Drazkowski JF, Wong WW, Fitch TR and Nelson KD: Metastatic prostate carcinoma mimicking meningioma: Case report and review of the literature. Neurologist 12: 48-52, 2006.

31. Lippman SM, Buzaid AC, Iacono RP, Steinbronn DV, Stanisic TH, Rennels MA, Yang PJ, Garewal HS and Ahmann FR: Cranial metastases from prostate cancer simulating meningioma: Report of two cases and review of the literature. Neurosurgery 19: 820-823, 1986

32. Tagle P, Villanueva P, Torrealba G and Huete I: Intracranial metastasis or meningioma? An uncommon clinical diagnostic dilemma. Surg Neurol 58: 241-245, 2002.

33. Kirkwood JR, Margolis MT and Newton TH: Prostatic metastasis to the base of the skull simulating meningioma en plaque. Am J Roentgenol Radium Ther Nucl Med 112: 774-778, 1971.

34. Fink LH: Metastasis of prostatic adenocarcinoma simulating a falx meningioma. Surg Neurol 12: 253-258, 1979.

35. Wong A, Koszyca B, Blumbergs PC, Sandhu N and Halcrow S: Malignant melanoma metastatic to a meningioma. Pathology 31: 162-165, 1999.

36. Jun P, Garcia J, Tihan T, McDermott MW and Cha S: Perfusion MR imaging of an intracranial collision tumor confirmed by image-guided biopsy. AJNR Am J Neuroradiol 27: 94-97, 2006.

37. Bulakbasi N, Kocaoglu M, Ors F, Tayfun C and Uçöz T: Combination of single-voxel proton MR spectroscopy and apparent diffusion coefficient calculation in the evaluation of common brain tumors. AJNR Am J Neuroradiol 24: 225-233, 2003.

38. Fukushima Y, Ota T, Mukasa A, Uozaki H, Kawai K and Saito N: Tumor to tumor metastasis: Lung adenocarcinoma metastasizing to vestibular schwannoma suspected on preoperative [18F]-fluorodeoxyglucose positron emission tomography imaging. World Neurosurg 78: 553.e9-553.e13, 2012. 